\title{
Optimal Temporal Gap between two Different Visual Stimuli in Perceptual Decision- Making
}

\section{Maryam Rafiei ${ }^{1}$, Azra Jahanitabesh ${ }^{2}$, Reza Ebrahimpour ${ }^{3 *}$}

${ }^{1}$ Institute for Cognitive Science Studies, Tehran, Iran

${ }^{2}$ Department of Psychology, University of California, Davis, USA

${ }^{3}$ Faculty of Computer Engineering, Shahid Rajaee Teacher Training University, Tehran, Iran

Introduction: We encounter different information or stimuli. However, the combination of these stimuli and the quality of their presentation can influence our perception and decision. Despite the importance of these combined stimuli to our judgments and decisions, it is not yet clear how the characteristics of these stimuli affect the decision- making processes. For example, it is not clear whether the time interval between the information we receive can affect the accuracy and speed of decision- making. This study aimed to investigate the effect of the time interval between two different visual stimuli on perceptual decisionmaking. Materials and Methods: According to psychophysical experiments, it was possible to measure the response to perceptual stimuli and compare perceptual choices. In a Random Dot Motion (RDM), the task was displayed to the participants as the primary visual stimulus and a graphic cue as the second visual stimulus, at different intervals, and then the participant's decision accuracy and reaction time to each of these two stimuli were recorded and analyzed. Results: We found that in RDM (primary stimulus), the accuracy of participants' decisions are not affected by the time interval between the presentation of two stimuli. Instead, the accuracy of the response to the Cue (second visual stimulus) decreases in long time intervals between two stimuli. Interestingly, if two stimuli are presented simultaneously, the decision about each of these stimuli is reasonably accurate; yet, the speed of the decision- making process is slower than when a person encounters two stimuli separated by a time interval. Conclusion: The human visual system can distinguish visual stimuli that are presented with a short time interval in between; however, in exchange for correctly identifying these stimuli, the speed of decision- making may be slowed down.

*Corresponding Author: Reza Ebrahimpour

Email: rebrahimpour@sru.ac.ir 


\title{
فاصله زمانى بهينه بين دو محرك متفاوت ديدارى در تصميم گيرى ادراكى
}

\author{
مريم رفيعى'، عذرا جهانى تابش'، رضا ابر اهيم يور”"” \\ 'موسسه آموزش عالى علوم شناختى، تهران، ايران

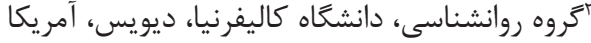

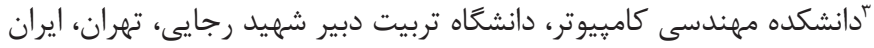

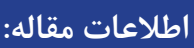

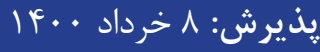

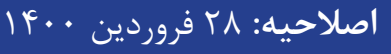

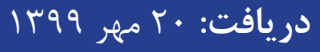

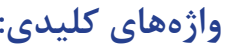

1 - تصميم كيرى

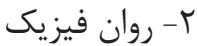

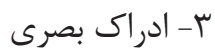

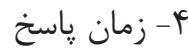

ه

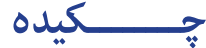

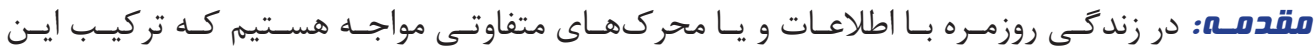

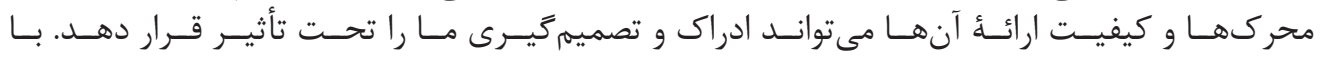

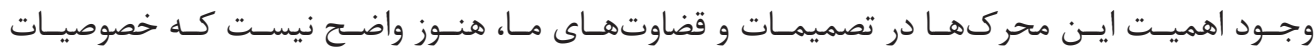

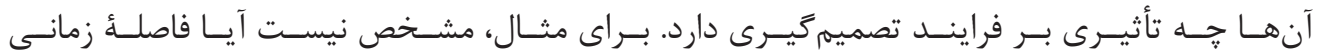

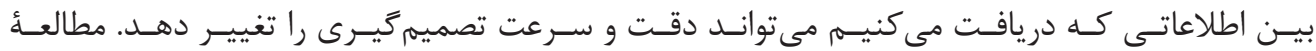

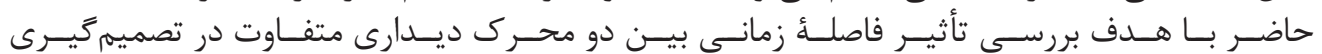

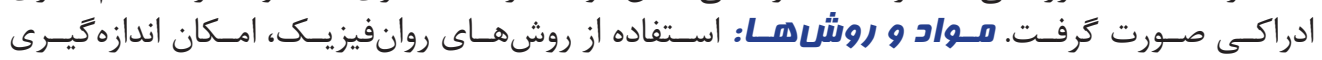

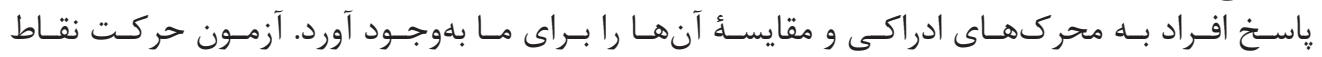

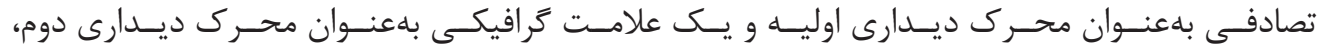

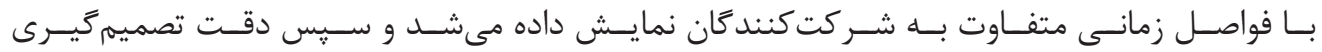

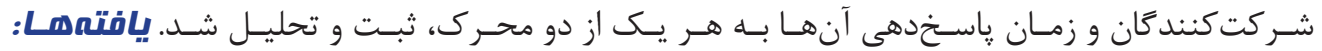

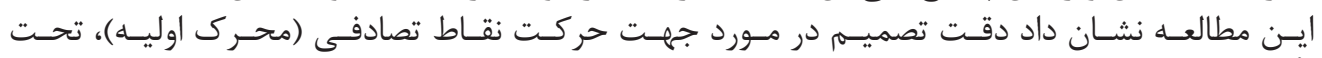

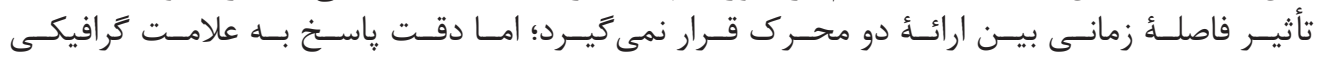

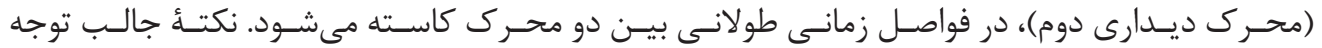

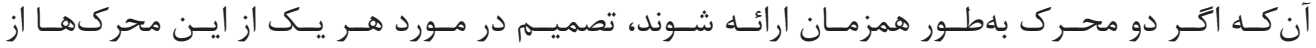

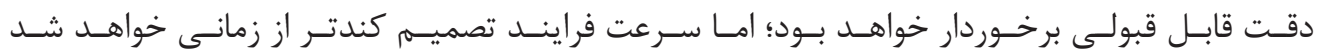

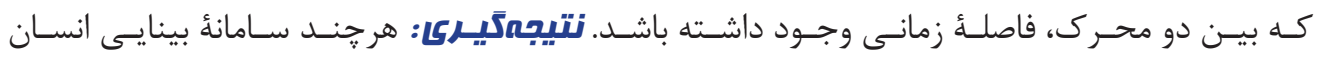

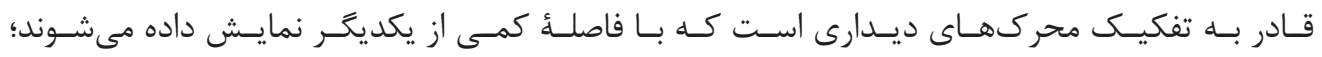

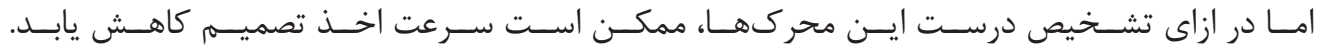




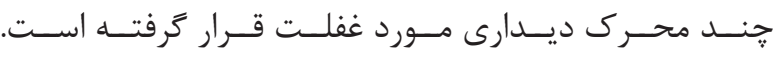

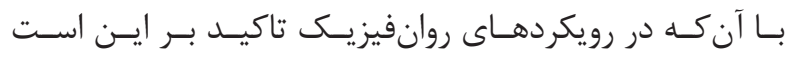

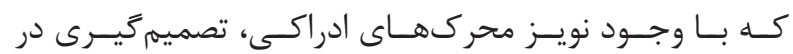

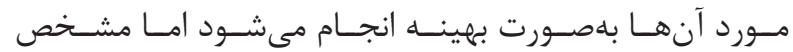

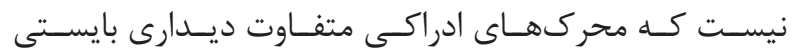

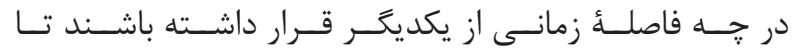

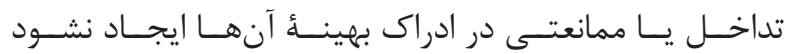

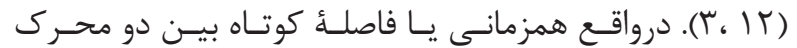

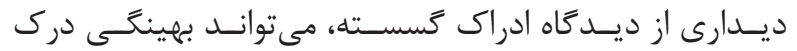

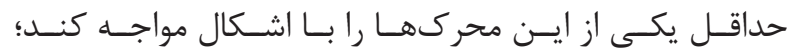

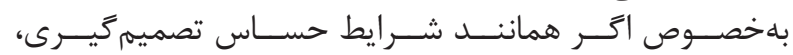

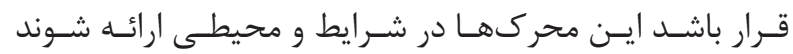

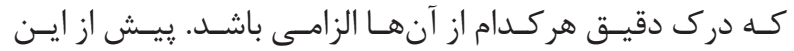

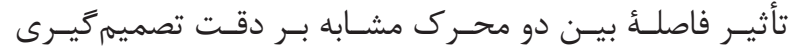

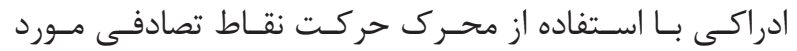

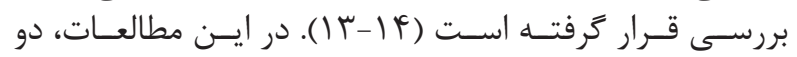

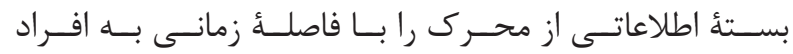

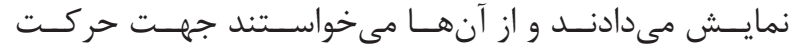

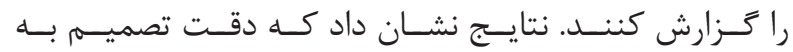

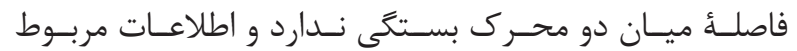

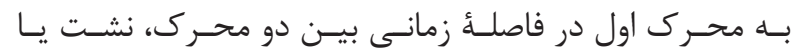

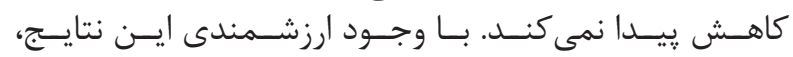

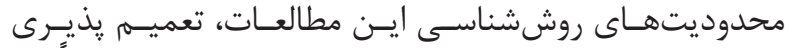

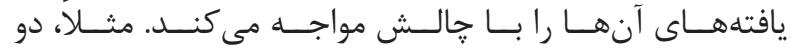

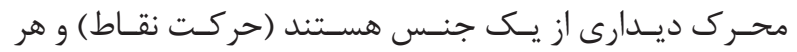

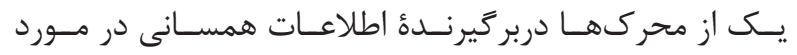

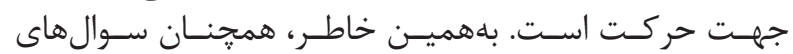

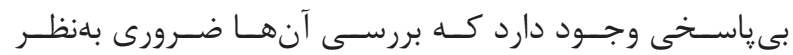

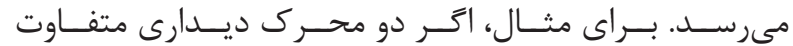

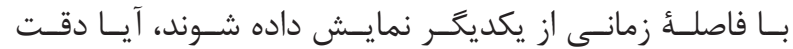

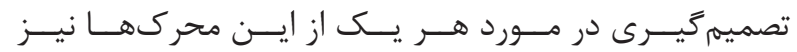

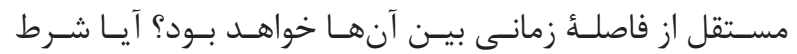

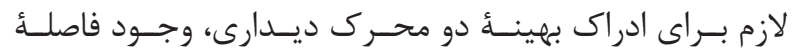

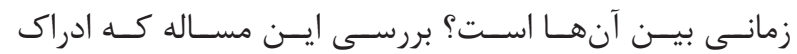

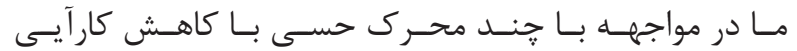

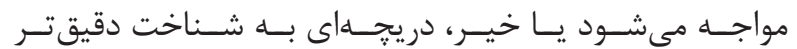

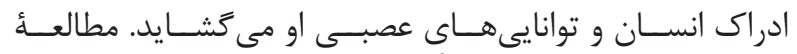

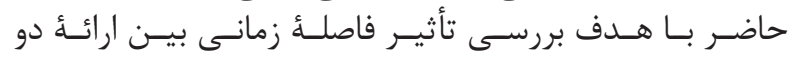

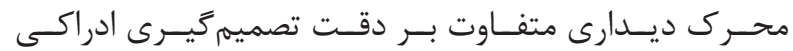

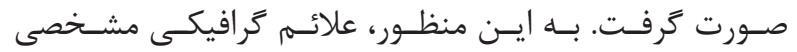

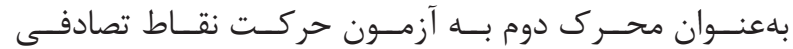

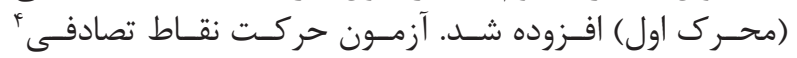

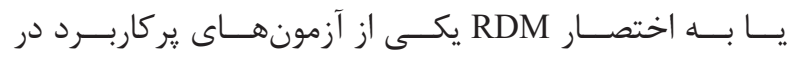

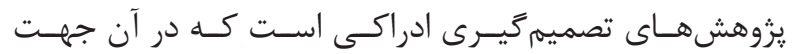

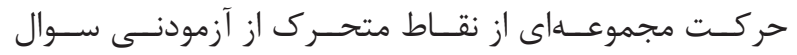

${ }^{1}$ Psychophysics

${ }^{2}$ Foveal vision
مقدمه

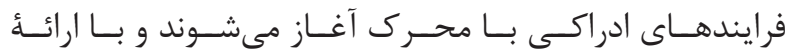

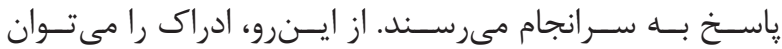

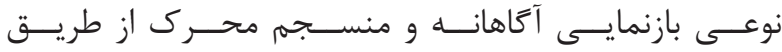

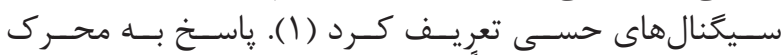

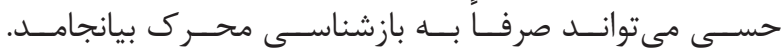

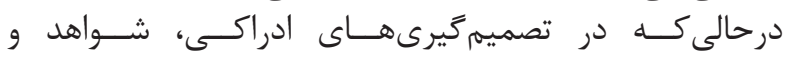

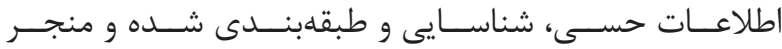

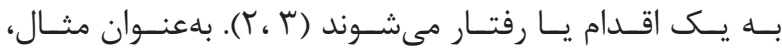

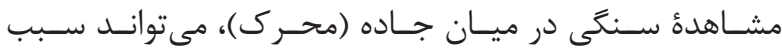

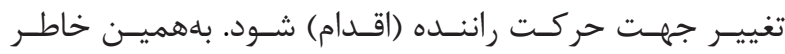

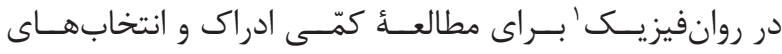

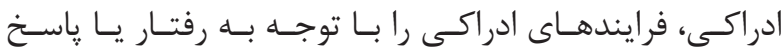

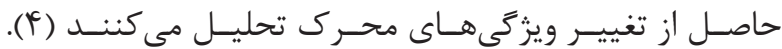

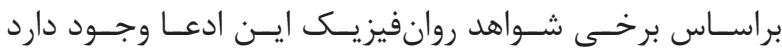

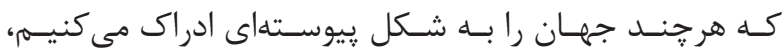

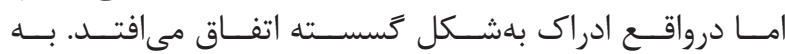

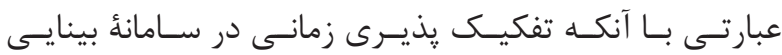

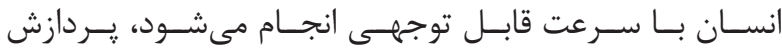

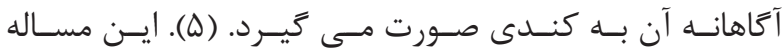

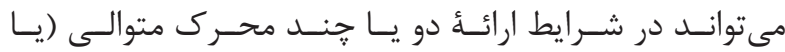

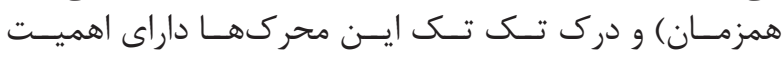

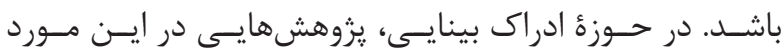

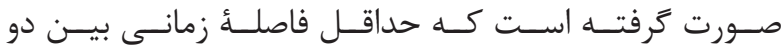

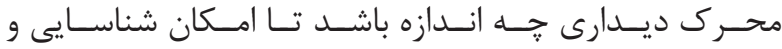

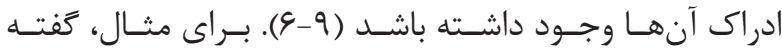

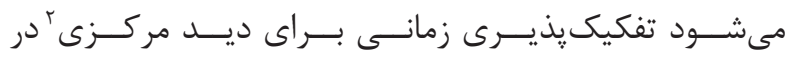

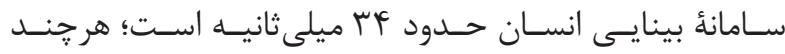

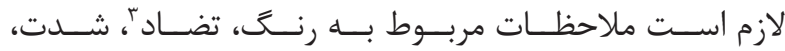

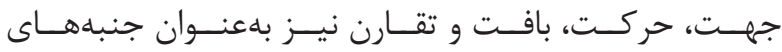

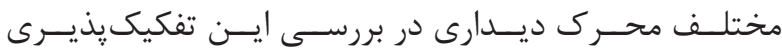

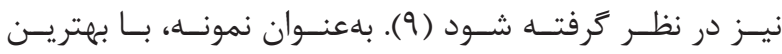

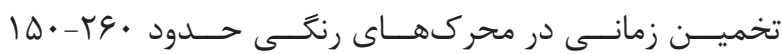

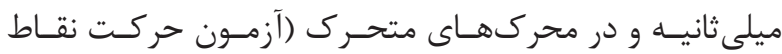

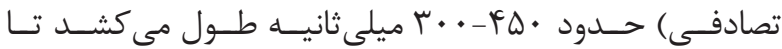

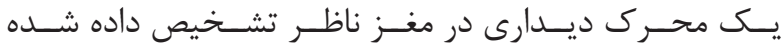

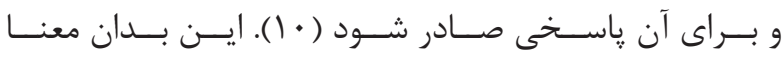

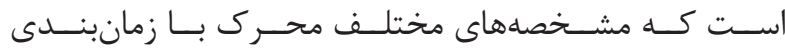

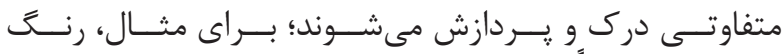

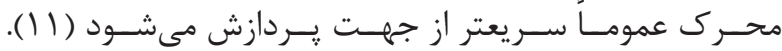

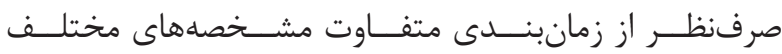

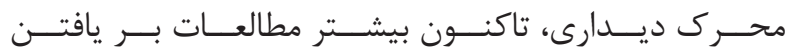

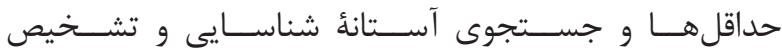

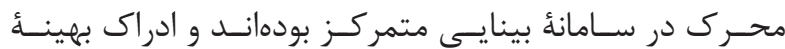

\footnotetext{
${ }^{3}$ Contrast

${ }^{4}$ Random Dot Motion
} 


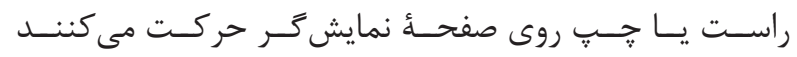

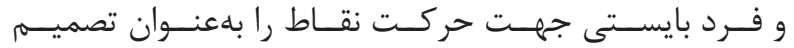

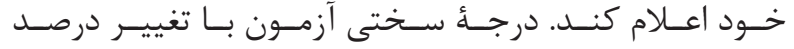

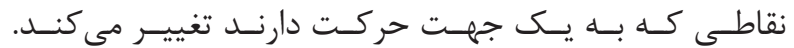

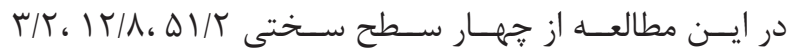

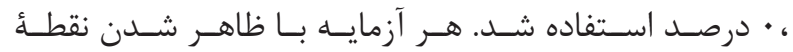

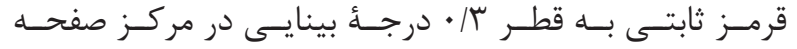

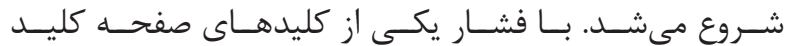

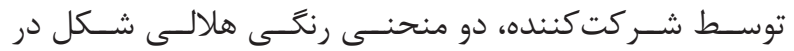

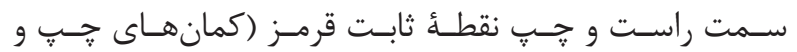

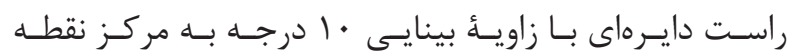

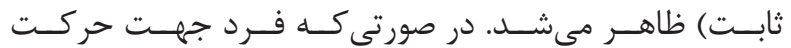

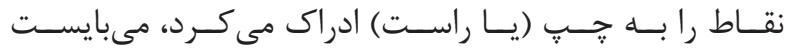

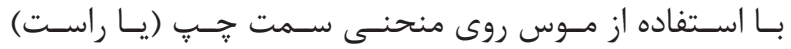

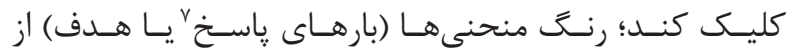

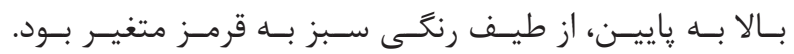

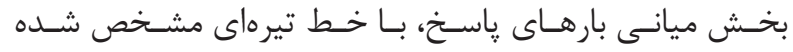

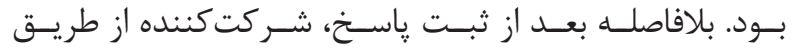

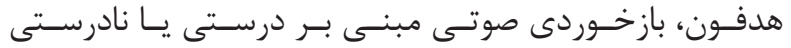

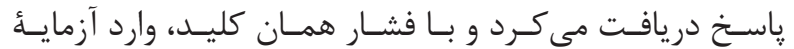

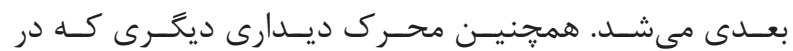

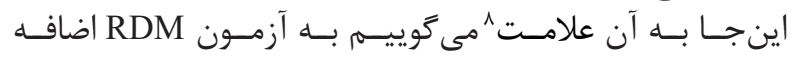

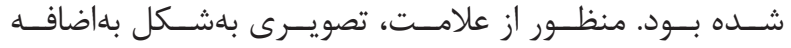

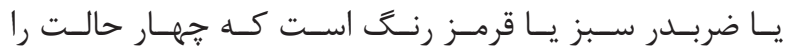

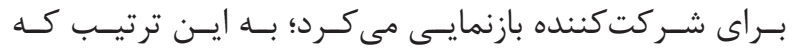

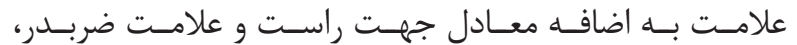

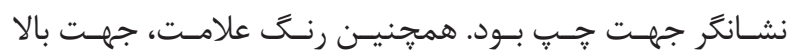

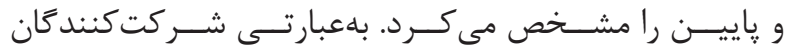

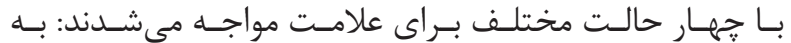

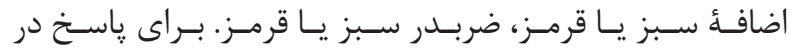

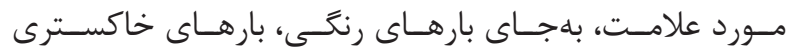

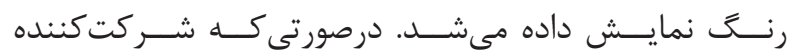

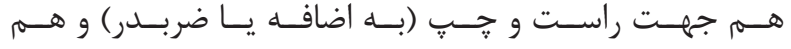

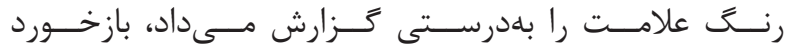

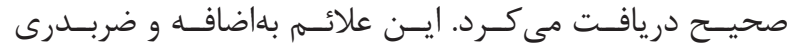

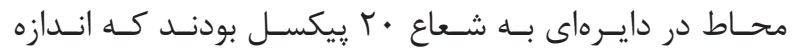

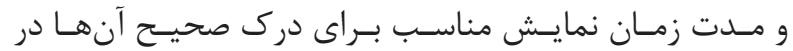

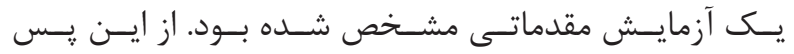

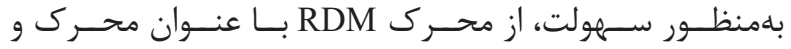

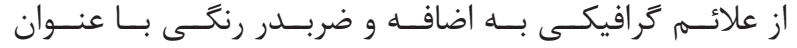

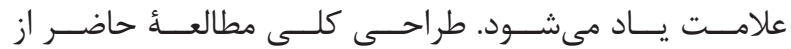

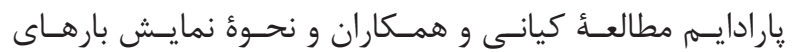

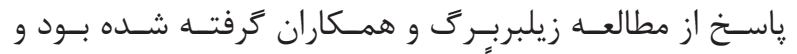

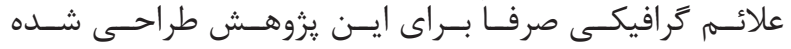

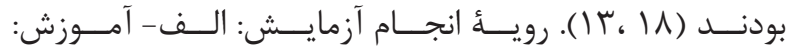

${ }^{5}$ Coherence

${ }^{6}$ Chinrest

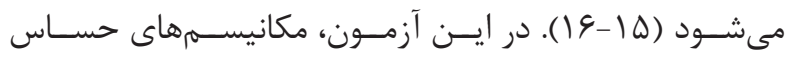

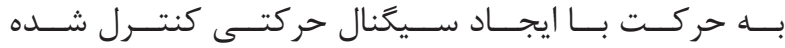

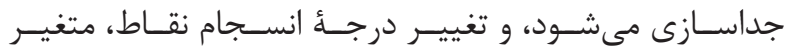

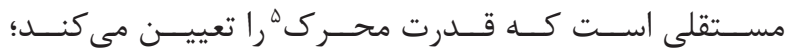

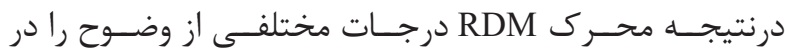

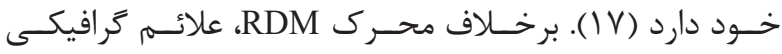

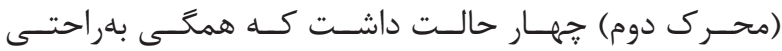

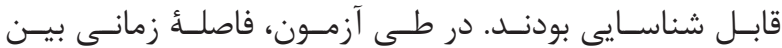

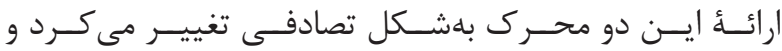

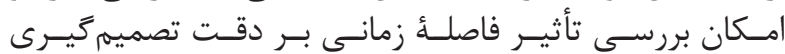

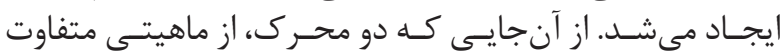

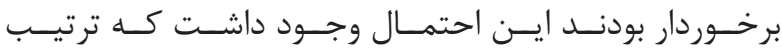

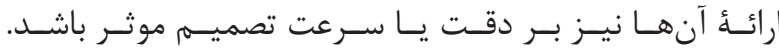

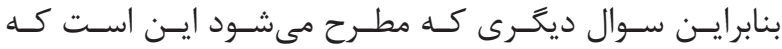

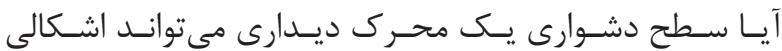

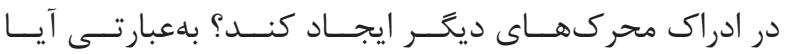

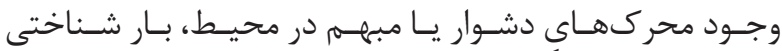

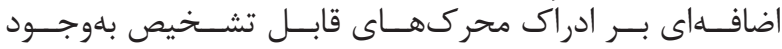

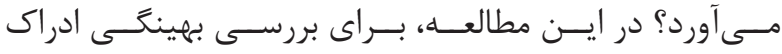

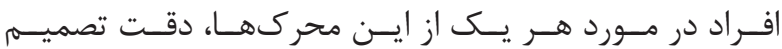

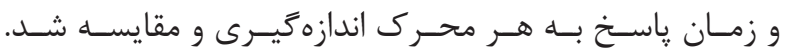

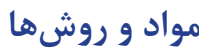

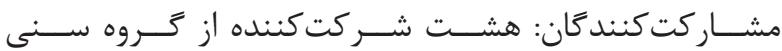

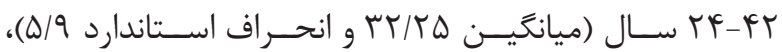

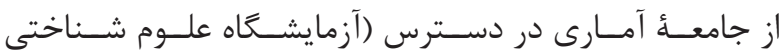

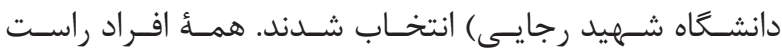

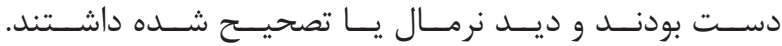

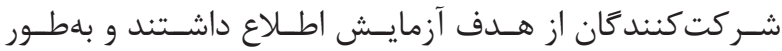

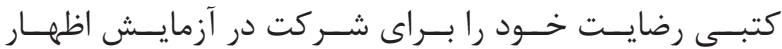

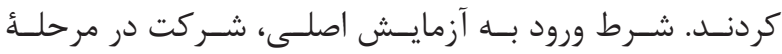

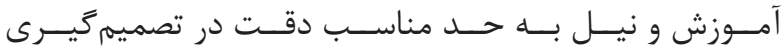

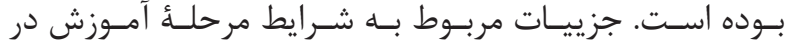

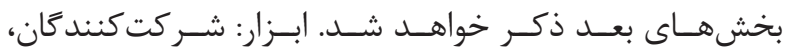

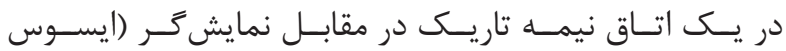

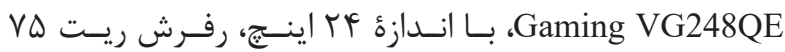

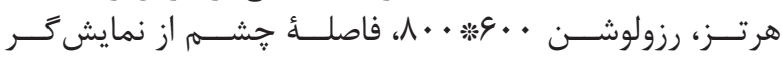

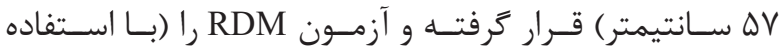

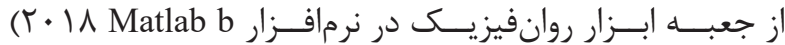

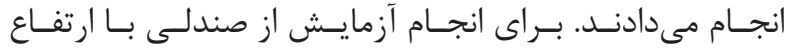

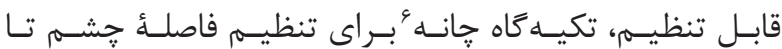

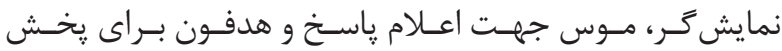

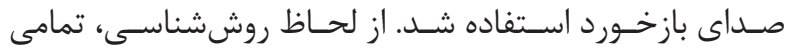

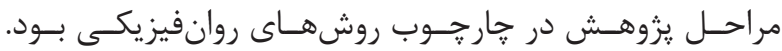

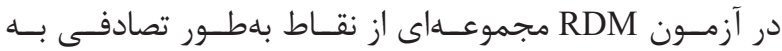

${ }^{7}$ Target

${ }^{8} \mathrm{Cue}$ 


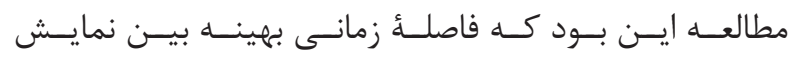

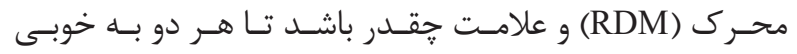

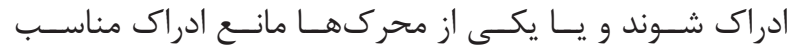

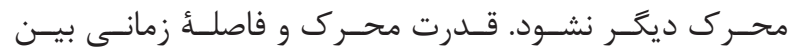

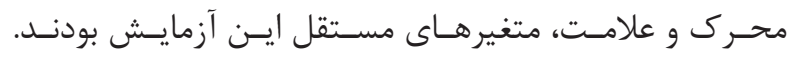

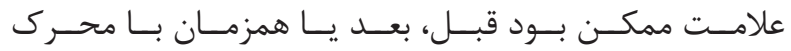

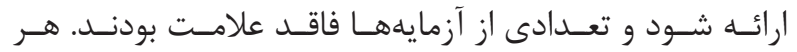

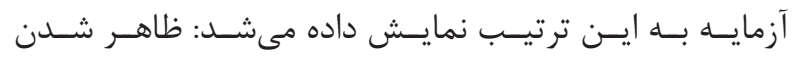

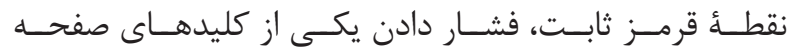

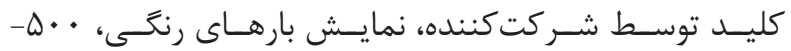

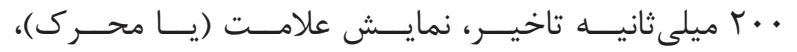

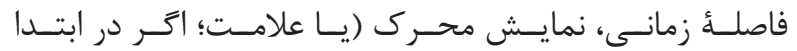

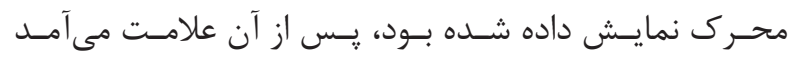

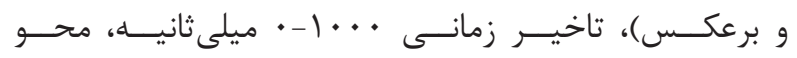

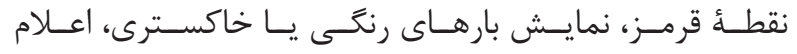

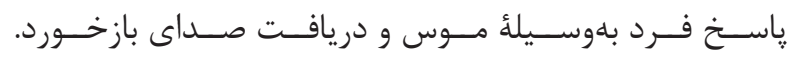

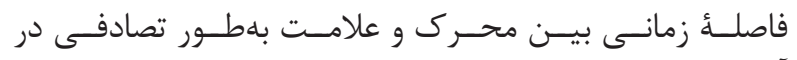

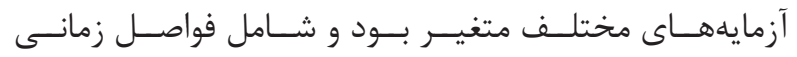

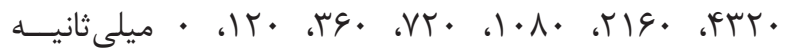

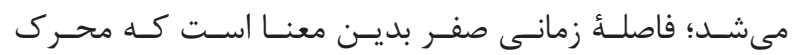

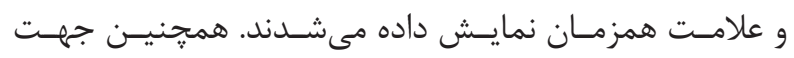

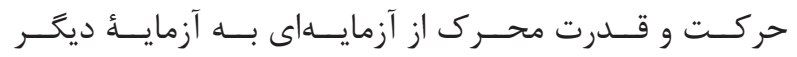

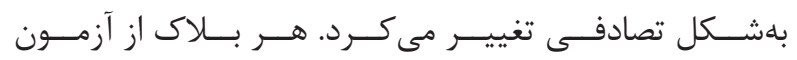

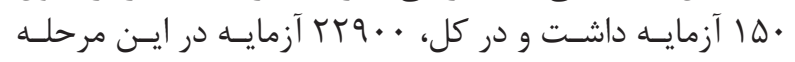

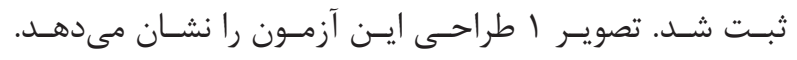

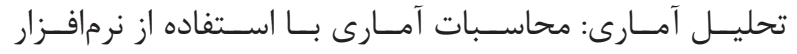
كatlab b

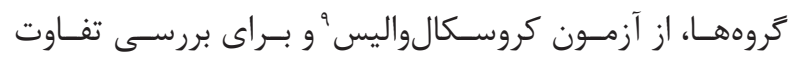

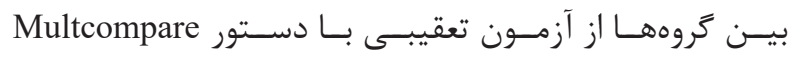

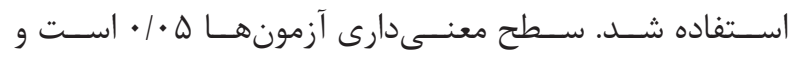

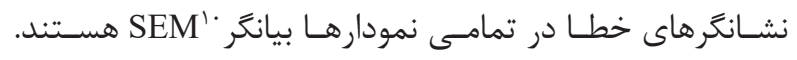

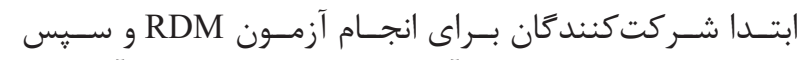

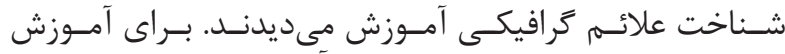

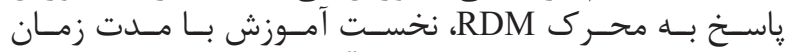

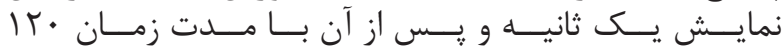

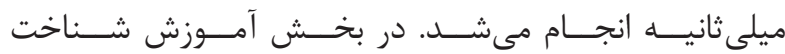

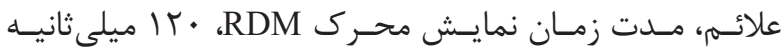

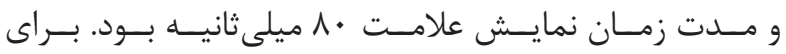

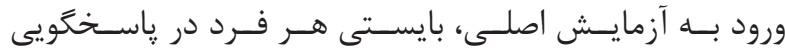

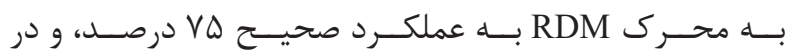

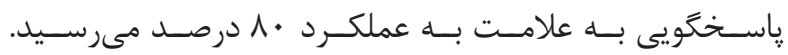

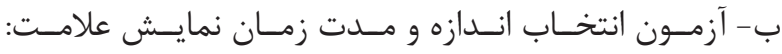

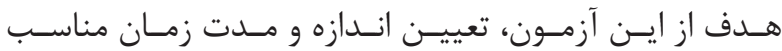

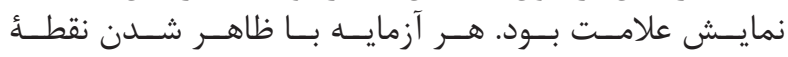

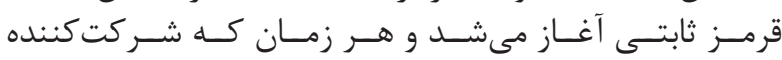

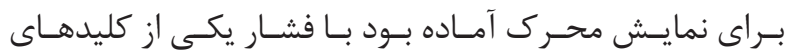

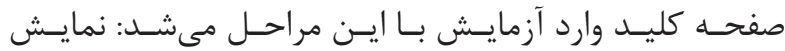

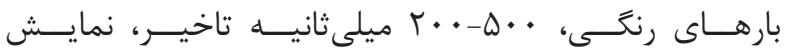

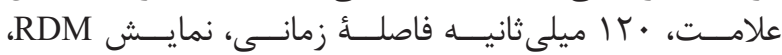

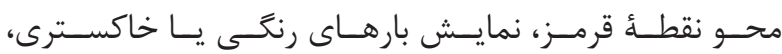

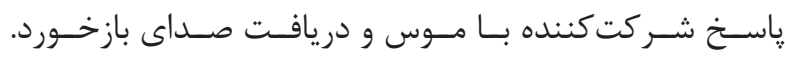

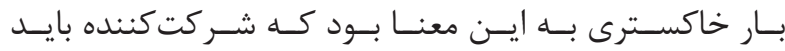

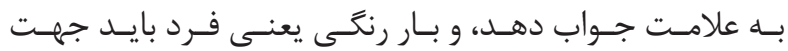

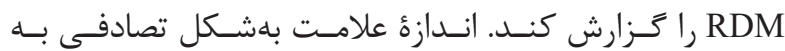

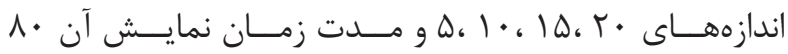

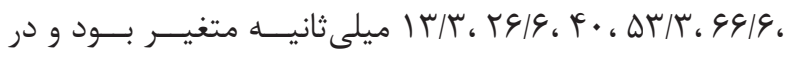

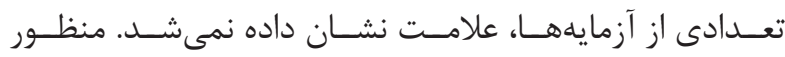

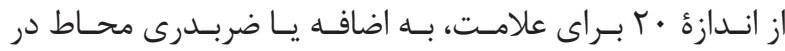

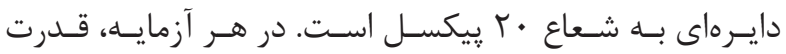

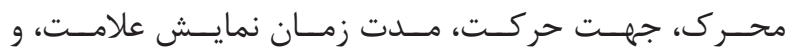

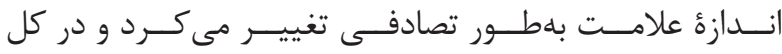

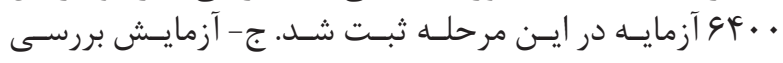

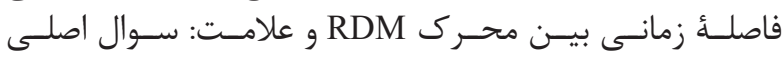

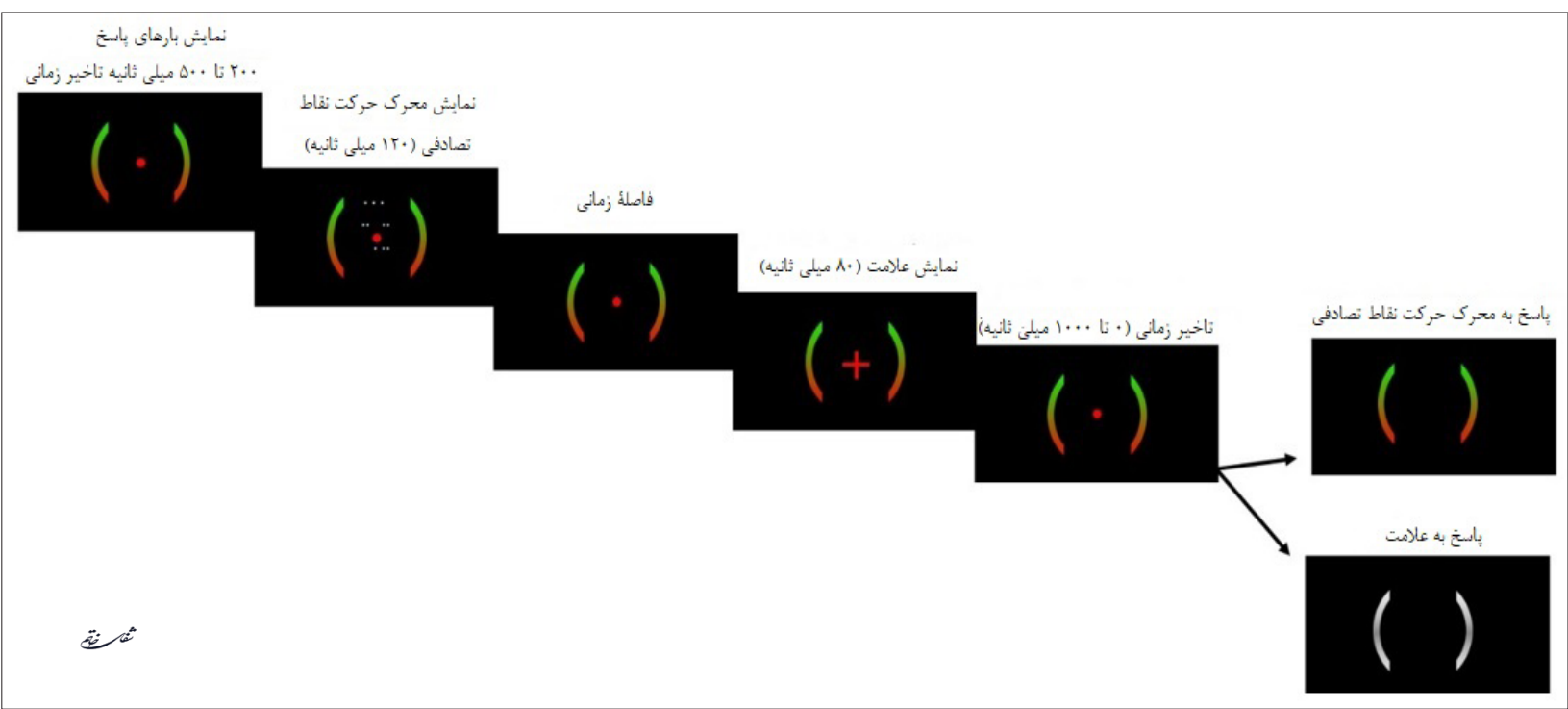

تصوير ا- طراحى آزمايش. در اين تصوير، علامت بعد از محرى RDM ارائه شده است، و در بايان، بارهاى رنكى (براى پاسخ به RDM) يا خاكسترى (براى باسخ بهعلامت) به نمايش در مى آيد. 


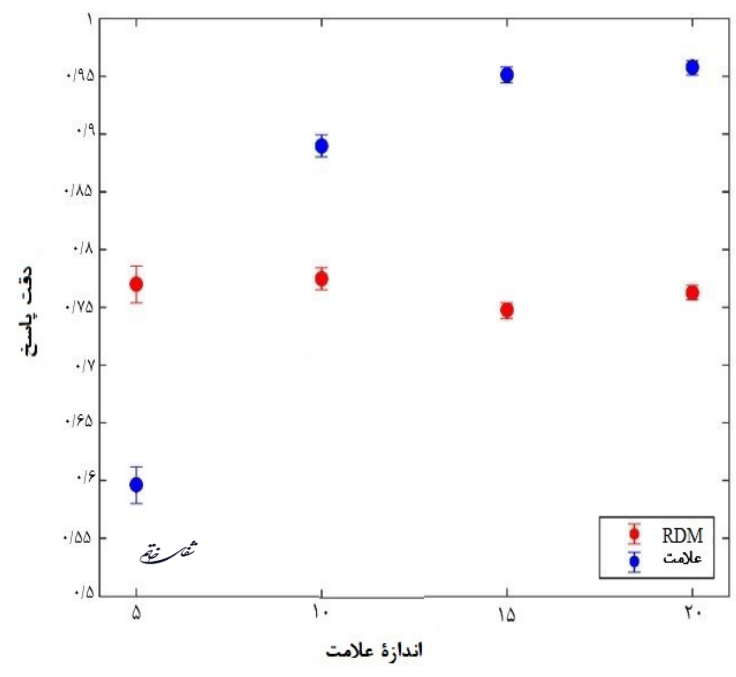

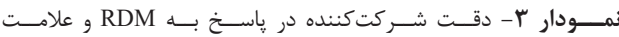

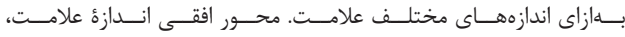

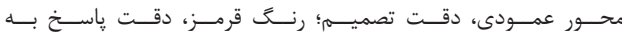

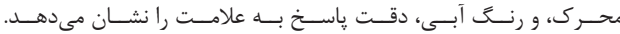

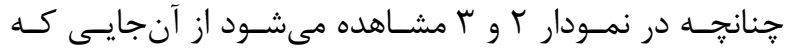

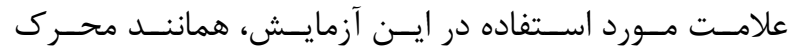

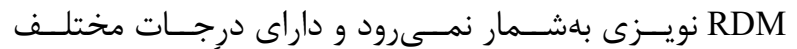

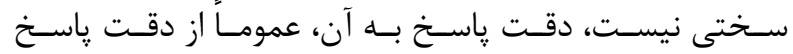

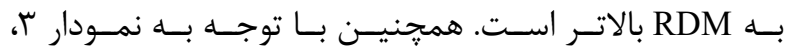

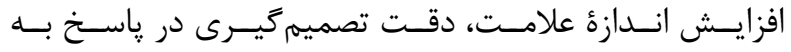

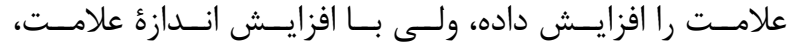

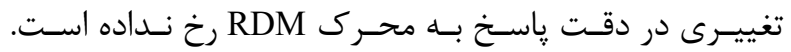

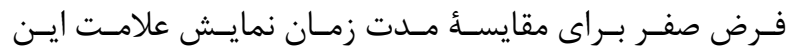

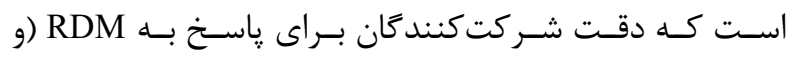

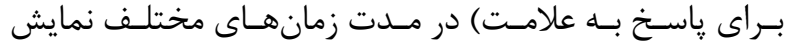

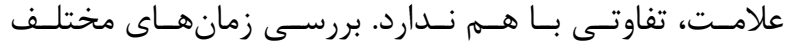

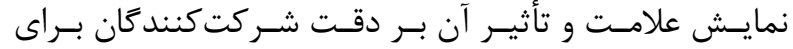

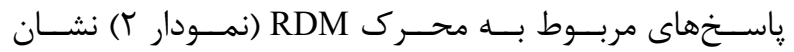

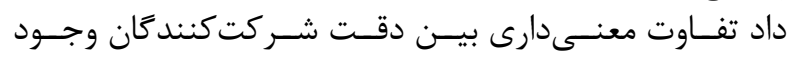

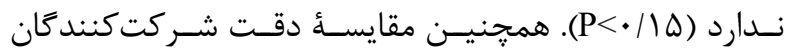

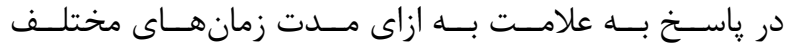

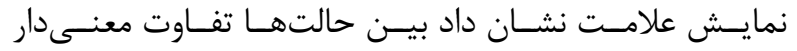

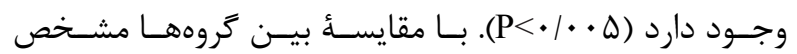

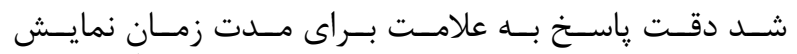

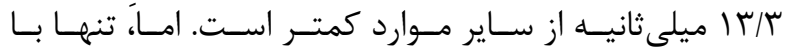

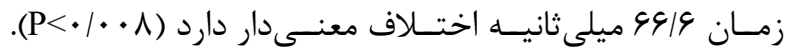

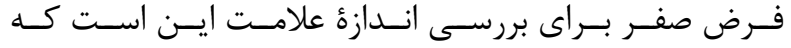

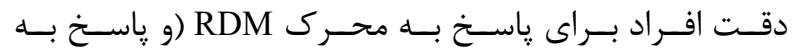

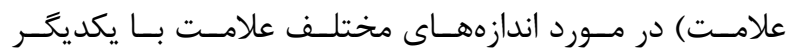

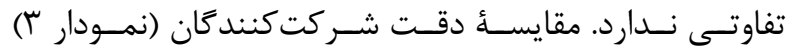

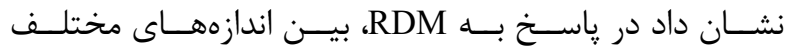

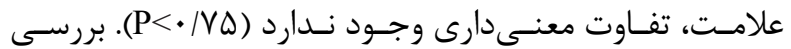

يافتهها

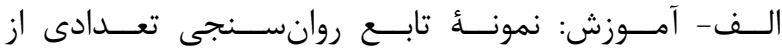

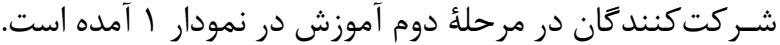

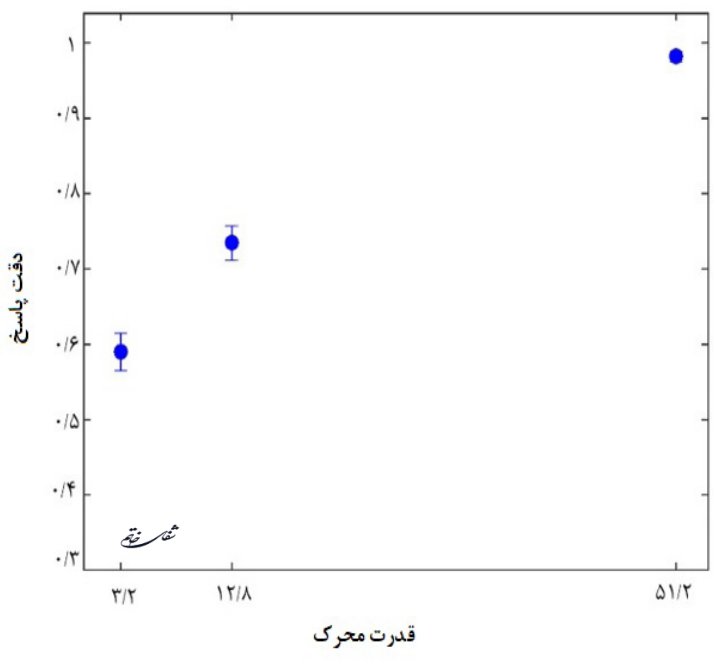

نمودار ا- تابع روانسنجى دقت شركت كندكان در مرحلة دوم آموزش.

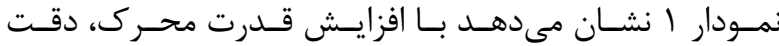

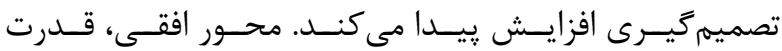

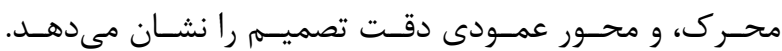

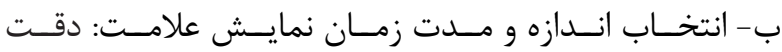

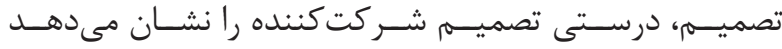

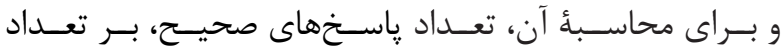

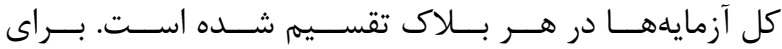

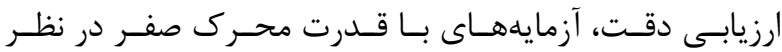

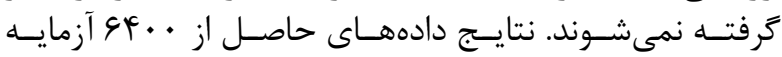

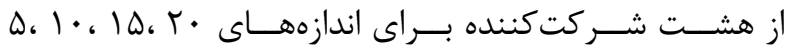

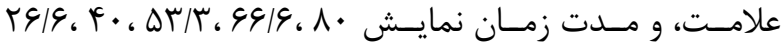

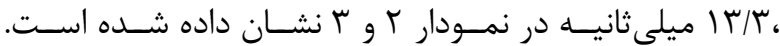

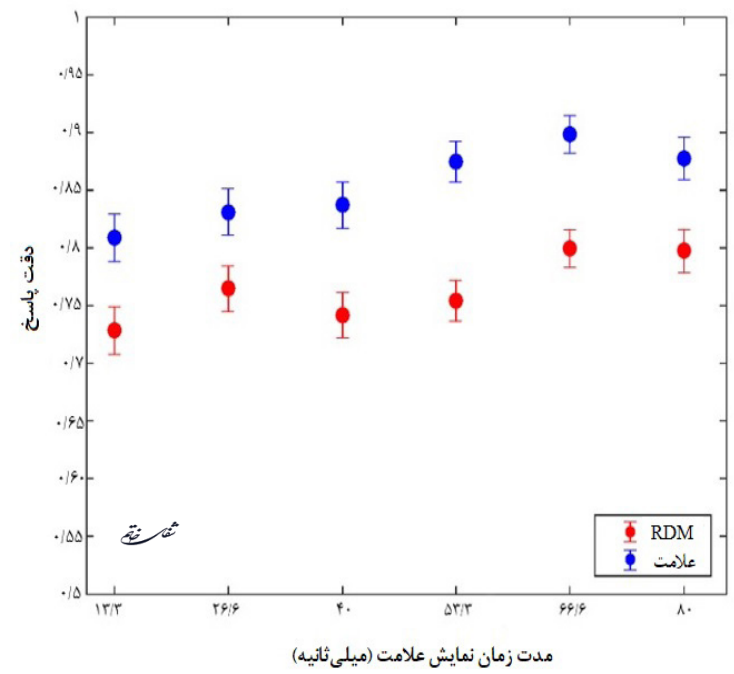

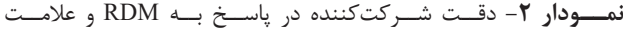

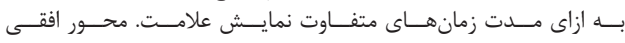

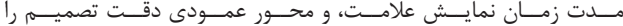

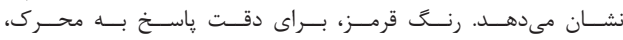

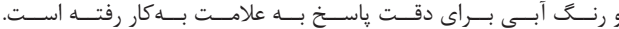




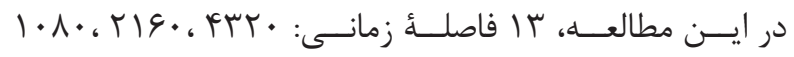

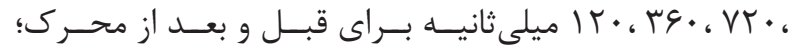

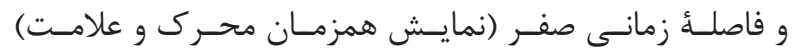

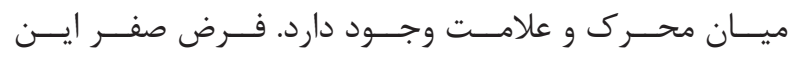

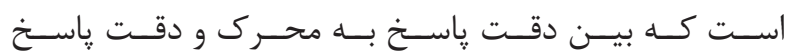

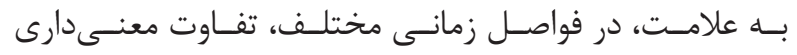

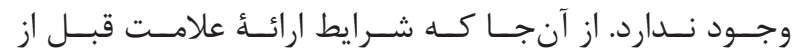

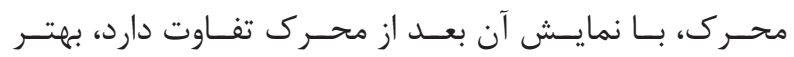

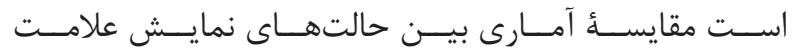

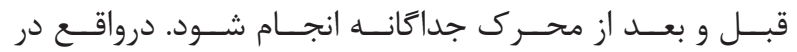

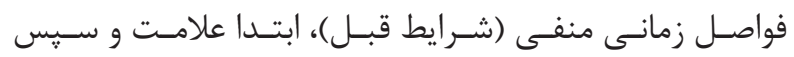

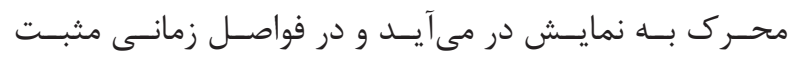

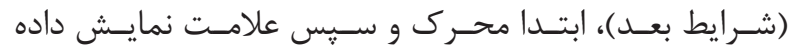

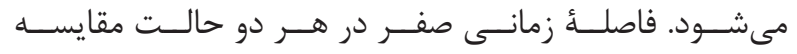

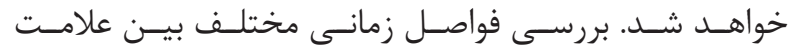

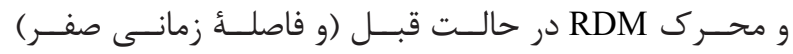

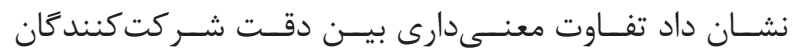

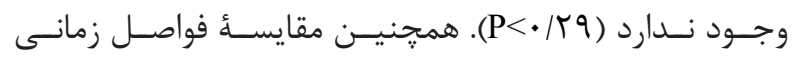

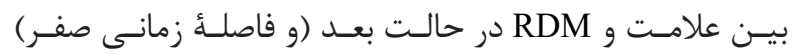

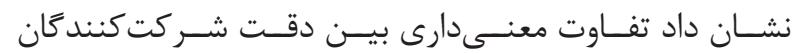

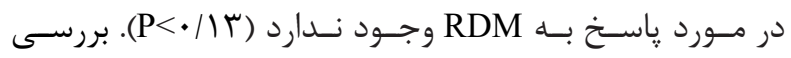

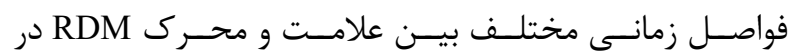

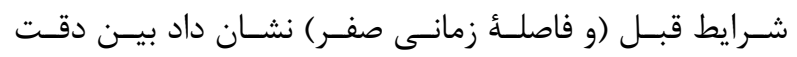

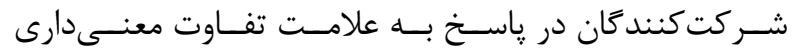

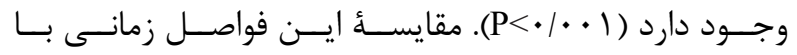

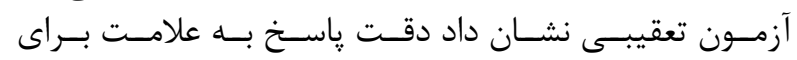

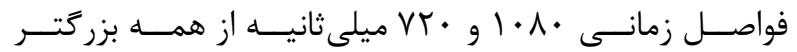

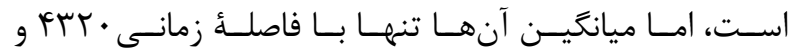

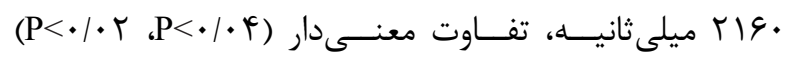

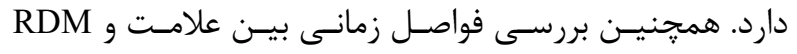

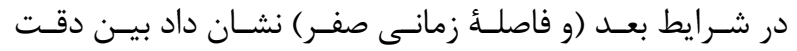

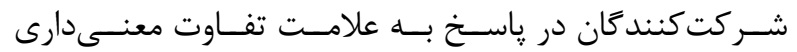

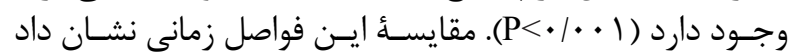

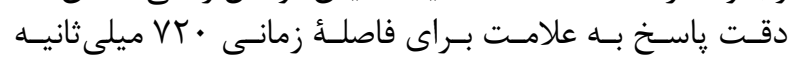

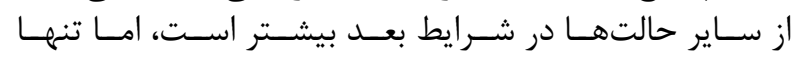

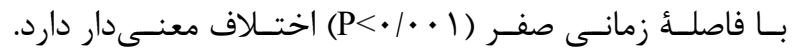

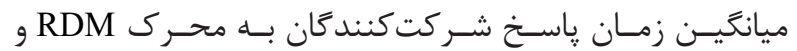

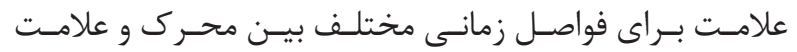

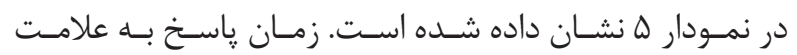

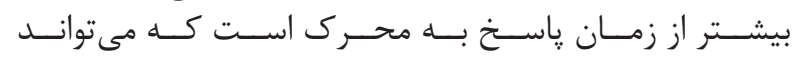

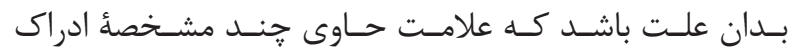

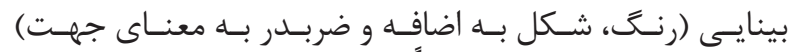

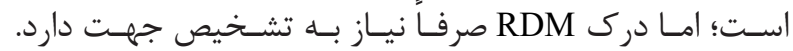

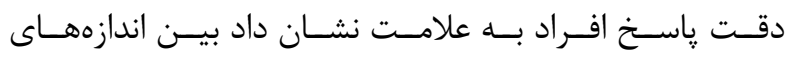

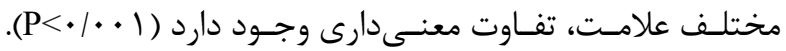

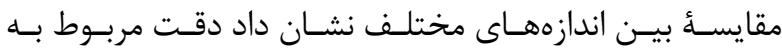

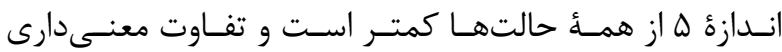

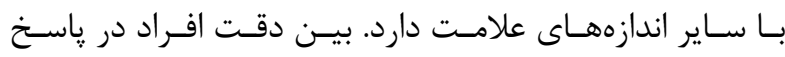

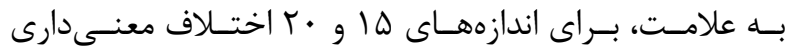

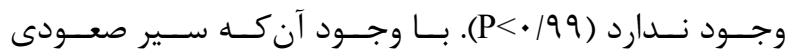

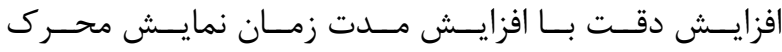

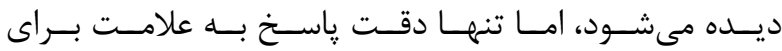

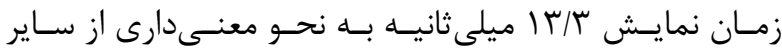

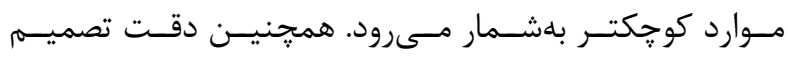

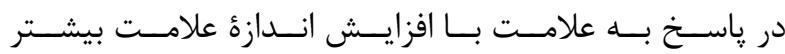

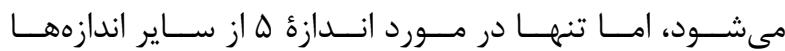

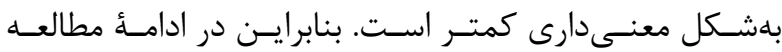

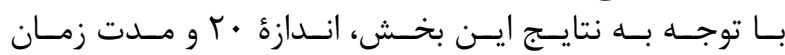

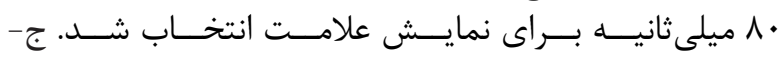

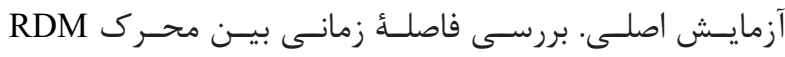
و علامـت: دقـت شــركت كنـند

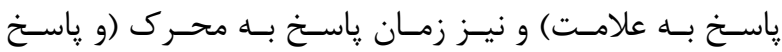

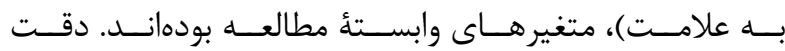

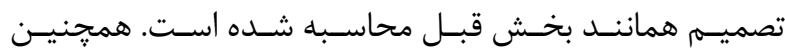

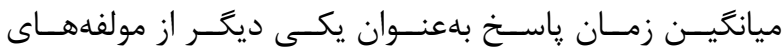

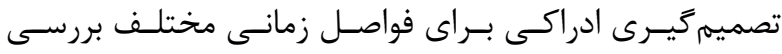

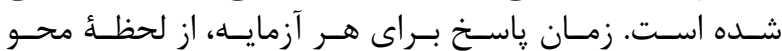

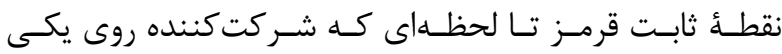

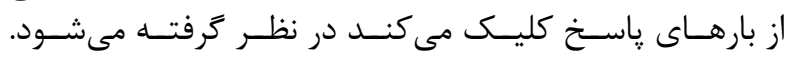

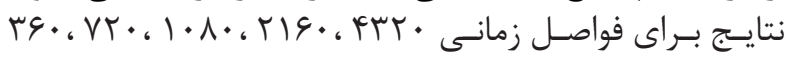

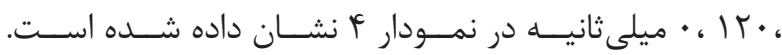

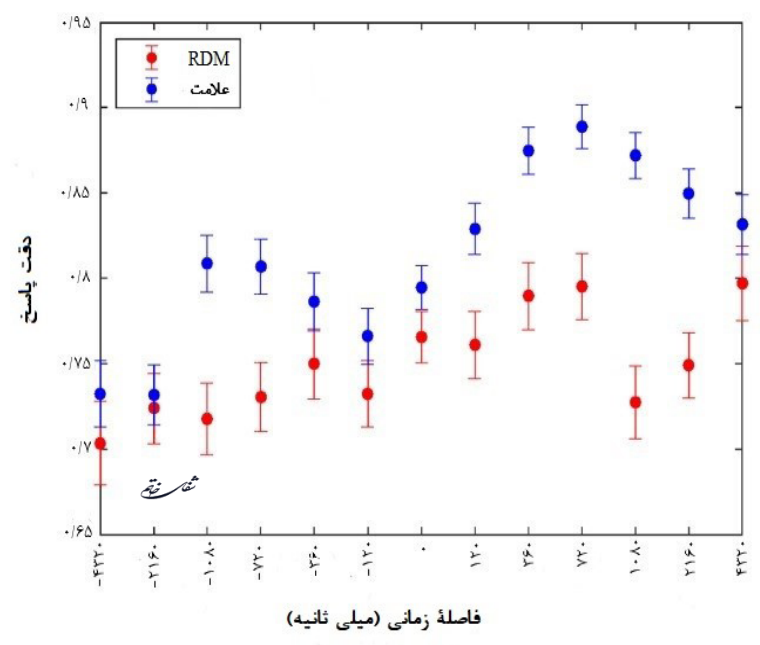

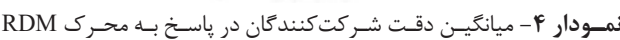

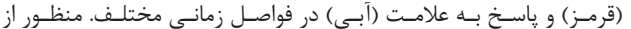

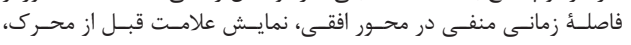

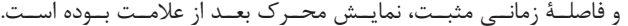

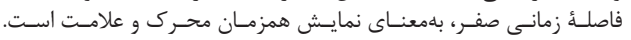




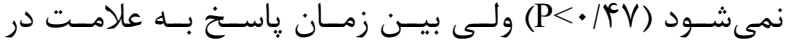

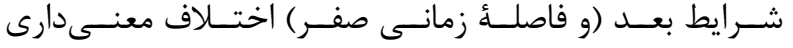

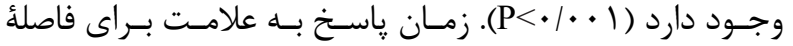

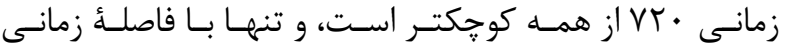

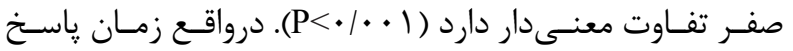

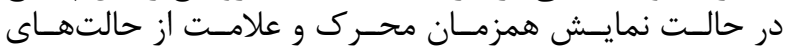

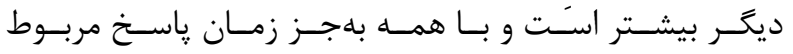

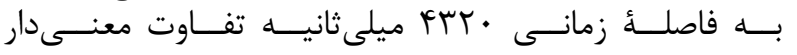

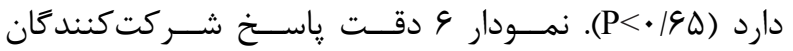

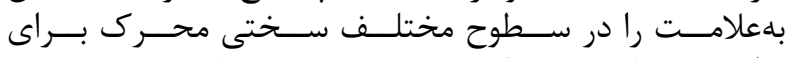

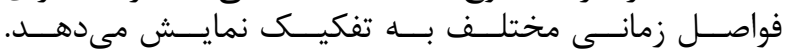

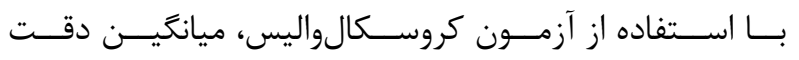

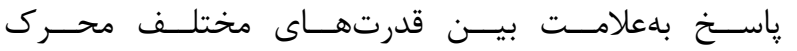

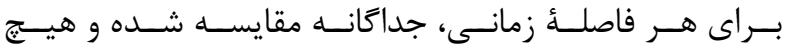

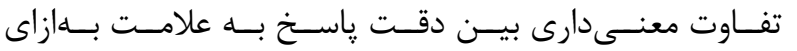

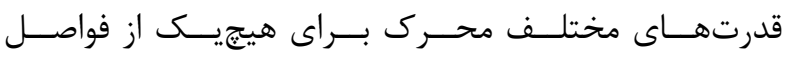

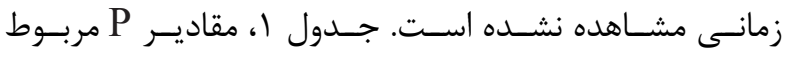

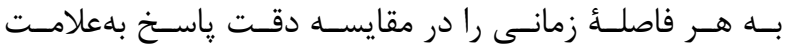

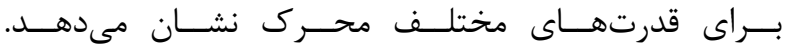

$$
\text { بحث و نتيجه }
$$

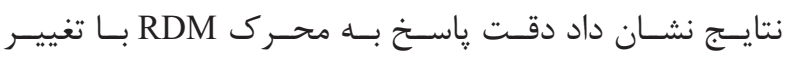

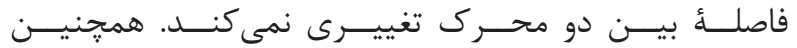

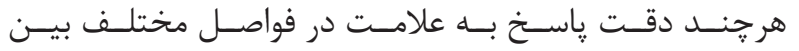

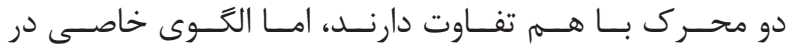

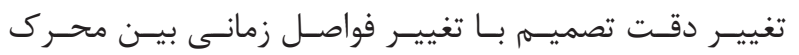

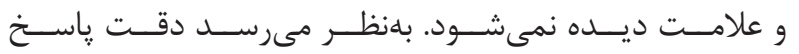

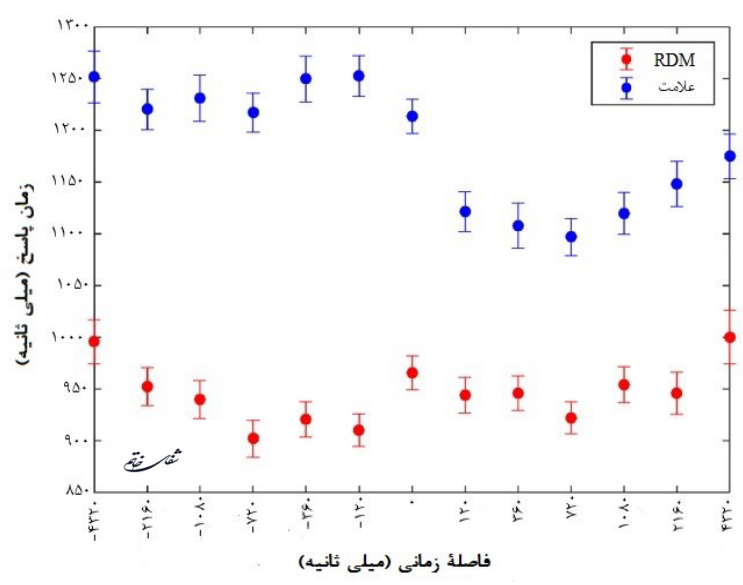

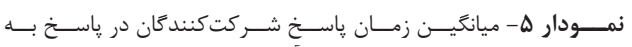

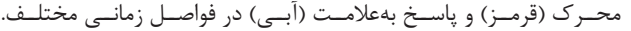

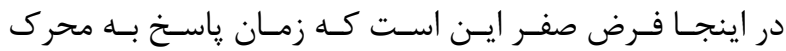

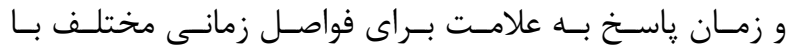

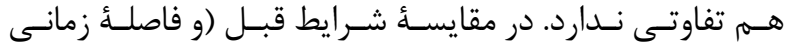

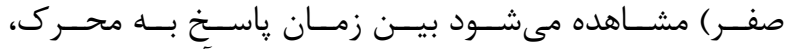

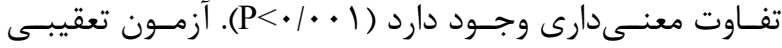

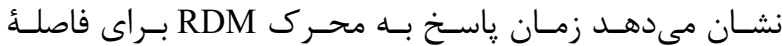

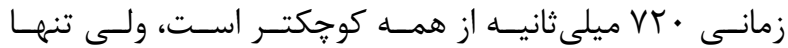

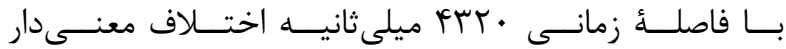

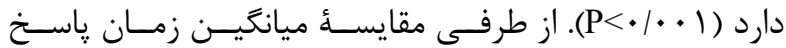

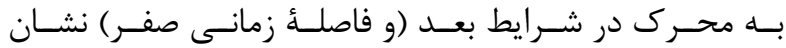

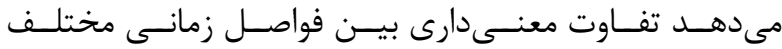

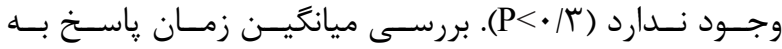

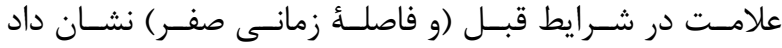

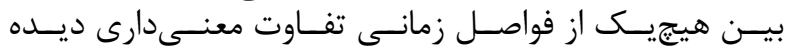

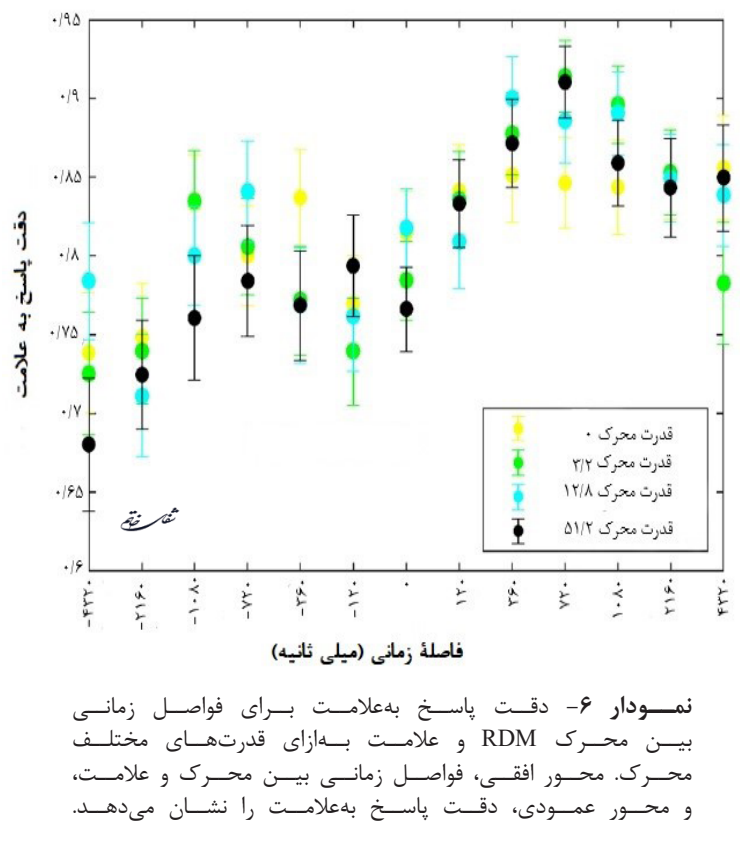

جدول ا-مقادير P براى مقايسٔ دقت پاسخ بهعلامت بهازاى قدرتهاى مختلف محرى RDM

\begin{tabular}{|c|c|c|c|c|c|c|c|c|c|c|c|c|c|}
\hline -Ftr. & -ris. & $-1 \cdot 1$. & -VT. & - TG. & -IT. & - & Ir. & ب. & VT. & $1 \cdot 1$. & ris. & Frt. & فاصلهُ زمانى \\
\hline זr/ & .1199 & $\cdot / 4 q$ & .191 & $\cdot|f|$ & $\cdot \mid V I$ & . & .19 & .181 & $\cdot / 4$ & .148 & .199 & ש & مقدار P \\
\hline
\end{tabular}




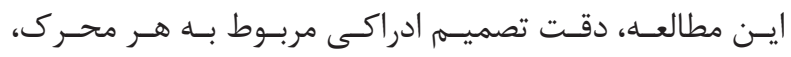

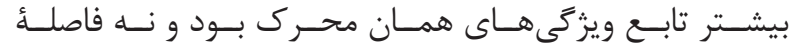

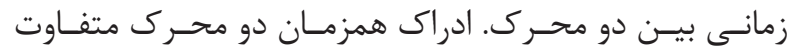

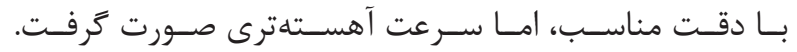

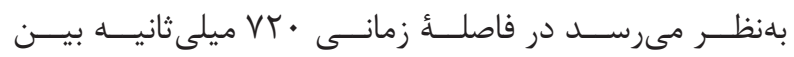

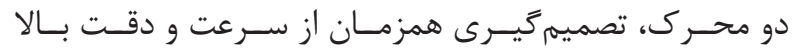

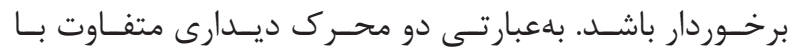

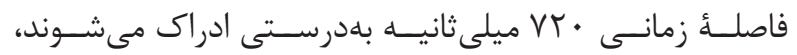

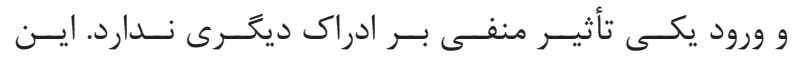

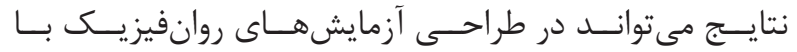

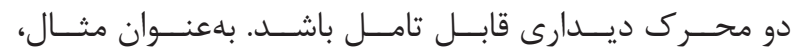

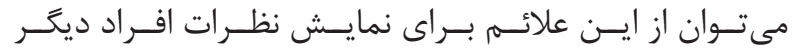

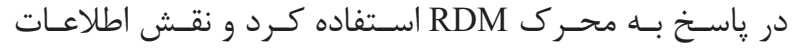

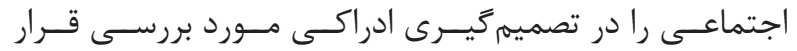

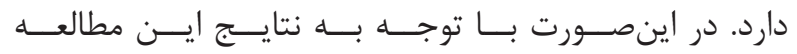

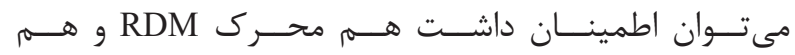

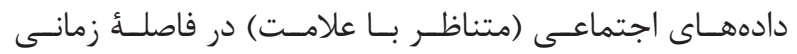

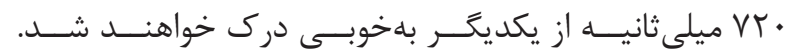

\section{تشكر و قدردانى}

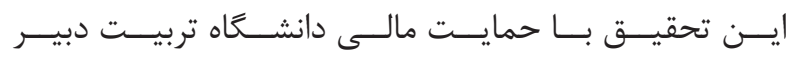

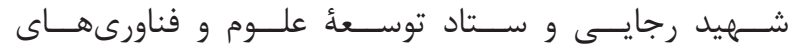

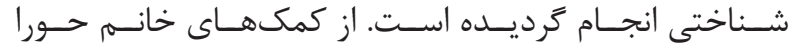

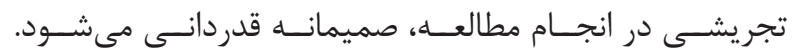

ملاحظات اخلاقى

اين يزوهش در دانشـعاه علوم يزشـكى ايران بررسى و با شناسه

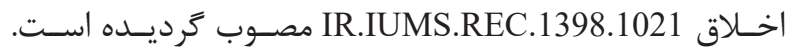

1. Goldstein EB, Brockmole J. Sensation and perception. Ninth ed: Cengage Learning; 2016. P. 5-8.

2. Hanks TD, Summerfield C. Perceptual decision making in rodents, monkeys, and humans. Neuron. 2017; 93(1): 15-31.

3. Summerfield C, Tsetsos K. Building bridges between perceptual and economic decisionmaking: neural and computational mechanisms. Frontiers in neuroscience. 2012; 6: 70

4. Bruce V, Green PR, Georgeson MA. Visual perception: Physiology, psychology, \& ecology. Psychology Press; 2003. p. 151-2.

5. Herzog MH, Kammer T, Scharnowski F. Time slices: what is the duration of a percept? PLoS Biology. 2016; 14(4): e1002433.

6. Arstila V, Georgescu AL, Pesonen H, Lunn D,

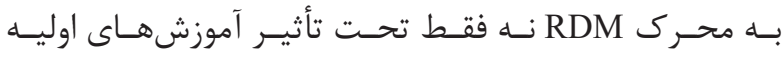

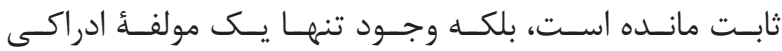

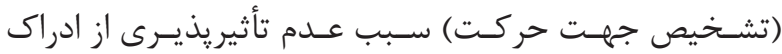

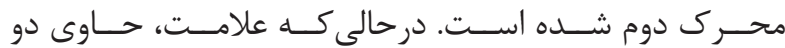

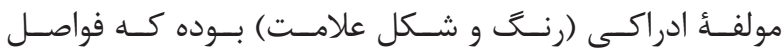

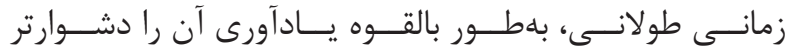

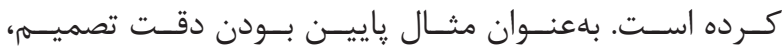

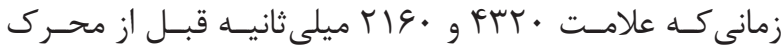

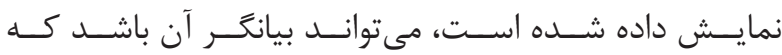

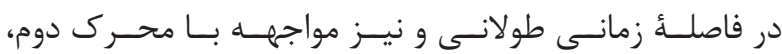

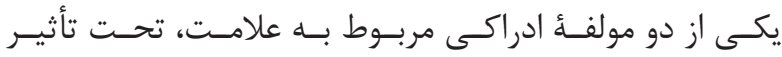

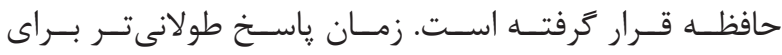

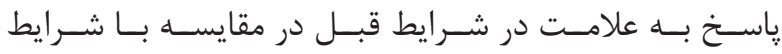

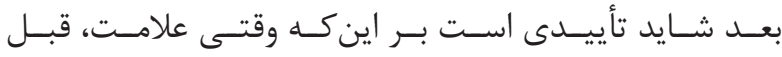

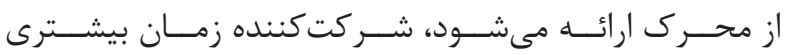

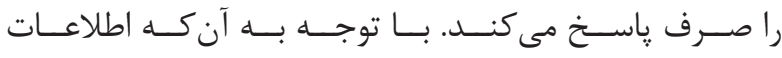

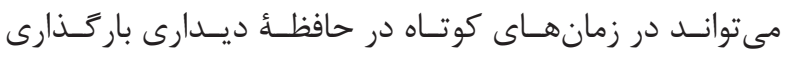

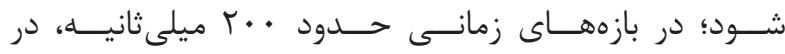

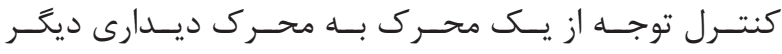

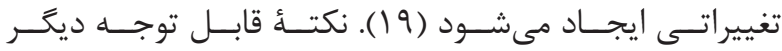

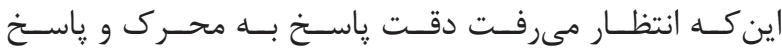

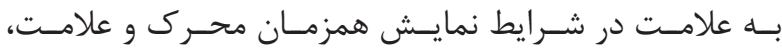

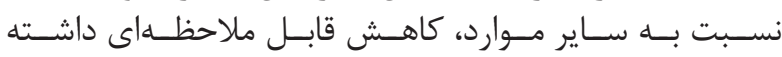

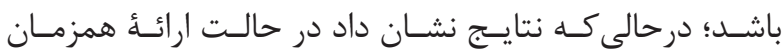

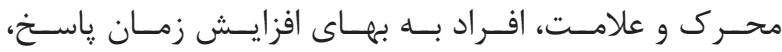

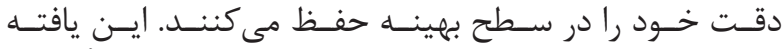

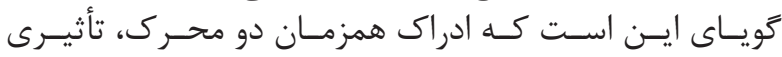

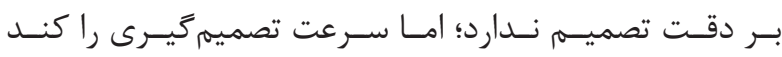

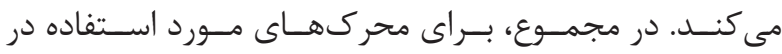

منابع human vision: Modulating factors and independent functions. PLoS One. 2020; 15(8): e0226122.

7. Poggel DA, Strasburger H. Visual perception in space and time-mapping the visual field of temporal resolution. Acta neurobiologiae experimentalis. 2004; 64(3): 427-37.

8. RutschmannR.Perception oftemporalorderandrelative visual latency. Science. 1966; 152(3725): 1099-101.

9. Reeves A. Temporal resolution in visual perception. Handbook of perception and action. 1: Elsevier; 1996. P. 11-24.

10. Amano K, Goda N, Nishida Sy, Ejima Y, Takeda $\mathrm{T}$, Ohtani Y. Estimation of the timing of human visual perception from magnetoencephalography. Journal of Neuroscience. 2006; 26(15): 3981-91. 
cued identification of orientation, color, and facial expressions. VPL-reports; 2020. P. 1-27.

\section{Rahnev D, Denison RN. Suboptimality in perceptual decision making. Behavioral and Brain Sciences. 2018; 41: 1-66.}

13. Kiani R, Churchland AK, Shadlen MN. Integration of direction cues is invariant to the temporal gap between them. Journal of Neuroscience. 2013; 33(42): 16483-9.

14. Tohidi-Moghaddam M, Zabbah S, Ebrahimpour R. The role of the primary information on importance of the last information in decision making. Neurosci J Shefaye Khatam. 2016; 4: 26-34.

15. Liu T, Pleskac TJ. Neural correlates of evidence accumulation in a perceptual decision task. Journal of neurophysiology. 2011; 106(5): 2383-98.
16. Shooshtari SV, Sadrabadi JE, Azizi Z, Ebrahimpour R. Confidence representation of perceptual decision by eeg and eye data in a random dot motion task. Neuroscience. 2019; 406: 510-27.

17. Britten KH, Shadlen MN, Newsome WT, Movshon JA. The analysis of visual motion: a comparison of neuronal and psychophysical performance. Journal of Neuroscience. 1992; 12(12): 4745-65.

18. Zylberberg A, Fetsch CR, Shadlen MN. The influence of evidence volatility on choice, reaction time and confidence in a perceptual decision. Elife. 2016; 5: e17688.

19. Beck VM, Hollingworth A, Luck SJ. Simultaneous control of attention by multiple working memory representations. Psychological science. 2012; 23(8): 887-98. 